\title{
Erratum to: Weighted subspace modeling for semantic concept retrieval using gaussian mixture models
}

\author{
Chao Chen ${ }^{1} \cdot$ Mei-Ling Shyu ${ }^{1} \cdot$ Shu-Ching Chen ${ }^{2}$
}

Published online: 23 July 2016

(C) Springer Science+Business Media New York 2016

Erratum to: Inf Syst Front (2016).

DOI 10.1007/s10796-016-9660-z

Due to a typesetting error, the biographies of Mei-Ling Shyu and Shu-Ching Chen were inadvertently switched. The original version has been corrected.

The online version of the original article can be found at http://dx.doi. org/10.1007/s10796-016-9660-z

Chao Chen

c.chen15@umiami.edu

Mei-Ling Shyu

shyu@miami.edu

Shu-Ching Chen

chens@cs.fiu.edu

1 Department of Electrical and Computer Engineering, University of Miami, 1251 Memorial Drive, Coral Gables, FL 33146, USA

2 School of Computing and Information Sciences, Florida International University, 11200 SW 8th Street, Miami, FL 33199, USA 